\title{
Capture Point: A Step toward Humanoid Push Recovery
}

\author{
Jerry Pratt, John Carff, Sergey Drakunov \\ Florida Institute for Human and Machine Cognition \\ Pensacola, Florida 32502 \\ Email: jpratt@ihmc.us
}

\author{
Ambarish Goswami \\ Honda Research Institute \\ Mountain View, California 94041 \\ Email: agoswami@honda-ri.com
}

\begin{abstract}
It is known that for a large magnitude push a human or a humanoid robot must take a step to avoid a fall. Despite some scattered results, a principled approach towards "When and where to take a step" has not yet emerged.

Towards this goal, we present methods for computing Capture Points and the Capture Region, the region on the ground where a humanoid must step to in order to come to a complete stop. The intersection between the Capture Region and the Base of Support determines which strategy the robot should adopt to successfully stop in a given situation.

Computing the Capture Region for a humanoid, in general, is very difficult. However, with simple models of walking, computation of the Capture Region is simplified. We extend the wellknown Linear Inverted Pendulum Model to include a flywheel body and show how to compute exact solutions of the Capture Region for this model. Adding rotational inertia enables the humanoid to control its centroidal angular momentum, much like the way human beings do, significantly enlarging the Capture Region.

We present simulations of a simple planar biped that can recover balance after a push by stepping to the Capture Region and using internal angular momentum. Ongoing work involves applying the solution from the simple model as an approximate solution to more complex simulations of bipedal walking, including a 3D biped with distributed mass.
\end{abstract}

\section{INTRODUCTION}

Push recovery is important for humanoid robots operating in human environments. No matter how well we attempt to shield these robots, it is inevitable that they will occasionally bump into objects and other people, and will be tripped up by debris, rocks, and objects that go undetected. Therefore, their ultimate utility will depend on good algorithms for push recovery.

Like most aspects of bipedal walking, push recovery is difficult because bipedal walking dynamics are high dimensional, non-linear, and hybrid. Moreover, a humanoid robot is underactuated and makes friction-limited unilateral contacts with the ground. Despite these theoretical difficulties, animals and humans are very adept at push recovery. As the push force progressively grows larger, strategies that they use include moving the Center of Pressure within the foot, accelerating internal angular momentum through lunging and "windmilling" of appendages, and taking a step. Although the humanoid literature contains several analysis and control techniques for each strategy, there is yet to emerge a principled approach towards "when and where to step" under a force disturbance.
This paper seeks to contribute in this direction using the concept of Capture Points.

A Capture Point is a point on the ground where the robot can step to in order to bring itself to a complete stop. A Capture Region is the collection of all Capture Points. Fast and accurate computation of Capture Points may be difficult and closed form solutions might not exist for a general humanoid robot. In this paper we examine simple models of walking that can be used to explain push recovery strategies, and to develop algorithms for implementing these strategies on humanoid robots. In particular, we study an extension to the well-known Linear Inverted Pendulum Model (LIP) [3], [4]. In this model the biped is approximated as a "hip" point mass which is maintained at a constant height, supported by a variable length leg link. For the Linear Inverted Pendulum Model there is a unique Capture Point corresponding to each state, for which we can obtain a closed-form expression.

Human movements such as a forward lunge and rapid arm rotations make use of angular momentum to maintain balance. However, the Linear Inverted Pendulum Model lacks rotational inertia and cannot capture this behavior. Therefore, we replace the point mass by a flywheel to explicitly model angular momentum about the body Center of Mass (CoM), resulting in the "Linear Inverted Pendulum Plus Flywheel Model" shown in Figure 1. By virtue of the ability to accelerate the Center of Mass by changing the angular momentum stored in the flywheel, the unique Capture Point extends to a set of contiguous points, the Capture Region.

During bipedal walking, trajectories in space do not need to be precise. In fact the only absolute constraints are usually to get from one point to another without falling down. Therefore, it is not critical that the feet be placed absolutely precisely. In addition, having relatively large feet and internal inertia provide additional control opportunities to make up for imprecise foot placement. Therefore, solutions for push recovery from the simple models we present can be successfully applied as approximations for controlling more complex bipeds. In ongoing work we are using the computations from the Linear Inverted Pendulum Plus Flywheel Model to control a three dimensional biped with distributed mass and feet, and without the constraint of a constant height Center of Mass. 


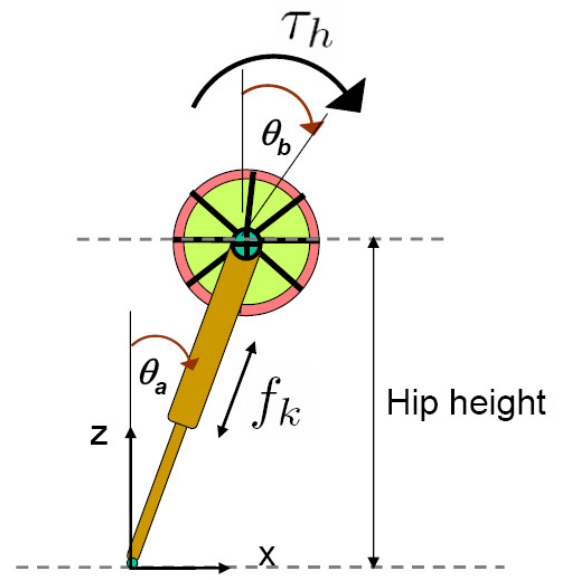

Fig. 1. Abstract model of a biped in the single support phase with a flywheel body and massless legs. The swing leg is not shown. The two actuators of the biped are located at the flywheel center (also the CoM of the biped) and the leg.

\section{BACKGROUND}

Human movements such as a forward lunge and rapid arm rotations make use of angular momentum to maintain balance. However, many models of bipedal walking, such as Kajita and Tanie's Linear Inverted Pendulum Model [3], treat the entire humanoid body as a point mass and do not incorporate the centroidal angular momentum, despite its significant influence in gait and balance [1], [5], [17].

There have been recent extensions to the Linear Inverted Pendulum Model that incorporate momentum. In particular, the Angular Momentum Pendulum Model (AMPM) [6], [7] is the closest to the model we present as the flywheel in our model can be seen as a physical embodiment of an angular momentum generator of the AMPM model.

To explicitly model centroidal angular momentum we choose a flywheel with centroidal moment of inertia and rotational angle limits. The flywheel (also called a reaction wheel) is a standard device used to control satellite orientation[15], [16]. Pratt [13] discussed the addition of a flywheel in simple biped models, but did not fully explore the models. Kuo and colleagues have demonstrated that humans use angular momentum to capture balance after disturbed through a "hip strategy" [8] and have pointed out the benefits of angular momentum for lateral stability in walking.

Although applicable to systems with actual physical flywheel devices, our approach to modeling the centroidal moment of inertia with a flywheel is intended for modeling bipeds with relatively limited rotation angles and velocities. For an example of using a traditional flywheel for the control of balance, refer to the work by Mayer, Farkas, and Asada [9].

Other related work includes the "resolved momentum control" scheme [5], in which a number of humanoid tasks can be controlled through the specification of linear and angular momenta and Hofmann's work [2] in which push recovery from significant disturbances is achieved using numerical optimization techniques.

\section{Capture Points and Push Recovery Strategy}

Using the concept of Capture Points and the Capture Region we can determine when and where to take a step to recover from a push:

- When to take a step: If a Capture Point is situated within the convex hull of the foot support area (the Base of Support), the robot is able to recover from the push without having to take a step, see Figure 2, top. Otherwise, it must take a step, see Figure 2, middle.

- Where to take a step: In order to stop in one step the robot must step such that its Base of Support regains an intersection with the Capture Region.

- Failure: The humanoid will fail to recover from a push in one step if the Capture Region in its entirety lies outside the kinematic workspace of the swing foot. In this case the robot must take at least two steps in order to stop, if it can stop at all. This is shown in Figure 2, bottom.

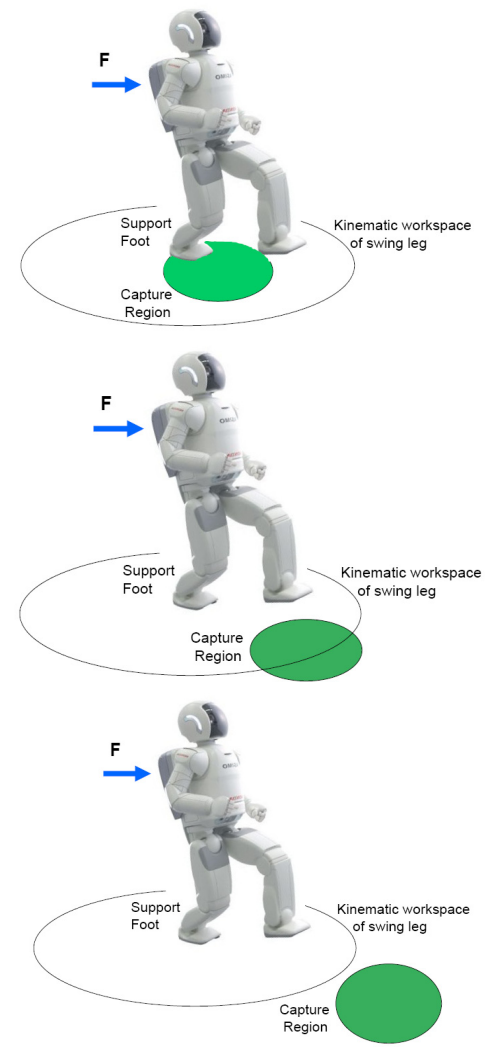

Fig. 2. When the Capture Region intersects the Base of Support, a humanoid can modulate its Center of Pressure to balance and does not need to take a step (top). When the Capture Region and Base of Support are disjoint, the humanoid must take a step to come to a stop (middle). If the Capture Region is outside the kinematic workspace of the swing foot, the humanoid cannot stop in one step (bottom figure).

The Capture Region makes it clear when and where a humanoid must step to in order to stop and therefore may lead to a more principled approach to humanoid push recovery. In the next Section we provide a more formal definition of Capture Points and Capture Regions. 


\section{Capture Points and Capture Regions}

We define a Capture State, a Safe Feasible Trajectory, and a Capture Point as follows:

Definition 1 (Capture State): State in which the kinetic energy of the biped is zero and can remain zero with suitable joint torques. Note that the Center of Mass must lie above the Center of Pressure in a Capture State. The vertical upright "home position"[1] is an example of a Capture State.

Definition 2 (Safe Feasible Trajectory): Trajectory through state space that is consistent with the robot's dynamics, is achievable by the robot's actuators, and does not contain any states in which the robot has fallen.

Definition 3 (Capture Point): For a biped in state $x$, a Capture Point, $P$, is a point on the ground such that if the biped covers $P$ (makes its Base of Support include $P$ ), either with its stance foot or by stepping to $P$ in a single step, and then maintains its Center of Pressure to lie on $P$, then there exists a Safe Feasible Trajectory leading to a Capture State.

The location of a Capture Point is dependent on the trajectory through state-space before and after swinging the leg and thus is not a unique point. Therefore, there exists a Capture Region such that if the Center of Pressure is placed inside this region, then the biped can stop for some state space trajectory.

Definition 4 (Capture Region): The set of all Capture Points.

For more information on Capture Points, including a discussion on their usefulness in defining stability margins for bipedal walking, please refer to [14]. While it is difficult to compute Capture Points for a general humanoid, we can easily compute them in closed form for some simplified models of walking. In the next Section we compute exact closedform solutions of the Capture Region for the Linear Inverted Pendulum Plus Flywheel Model.

\section{Computation of Capture Region for the Linear InVerted Pendulum Plus Flywheel Model}

\section{A. Equations of Motion of Planar Biped with Flywheel}

We begin with a biped system abstracted as a planar inverted pendulum with an inertial flywheel centered at the Center of Mass as shown in Figure 1. The legs of the biped are massless and extensible. The biped has two actuators located at the flywheel and the leg. The equations of motion during the single support phase are

$$
\begin{aligned}
m \ddot{x} & =f_{k} \sin \theta_{a}-\frac{\tau_{h}}{l} \cos \theta_{a} \\
m \ddot{z} & =-m g+f_{k} \cos \theta_{a}+\frac{\tau_{h}}{l} \sin \theta_{a} \\
J \ddot{\theta}_{b} & =\tau_{h}
\end{aligned}
$$

where $m$ and $J$ are the mass and rotational inertia of the flywheel, $g$ is the gravitational acceleration constant, $x$ and $z$ are the CoM coordinates, $l$ is the distance from the point foot to the CoM, $\theta_{a}$ and $\theta_{b}$ are respectively, the leg and the flywheel angles with respect to vertical, $\tau_{h}$ is the motor torque on the flywheel, and $f_{k}$ is the linear actuation force on the leg.

\section{B. Linear Inverted Pendulum Plus Flywheel Model}

The Linear Inverted Pendulum Plus Flywheel Model can be derived as a special case of the above model by setting $\dot{z}=0$ and $z=z_{0}$. From Equation 2 we can solve for $f_{k}$ as

$$
f_{k}=\frac{m g}{\cos \theta_{a}}-\frac{1}{l} \frac{\sin \theta_{a}}{\cos \theta_{a}} \tau_{h}
$$

Replacing $\cos \theta_{a}=\frac{z}{l}$ and $\sin \theta_{a}=\frac{x}{l}$, we get

$$
f_{k}=\frac{m g}{z_{0}} l-\frac{1}{l} \frac{x}{z_{0}} \tau_{h}
$$

Substituting $f_{k}$ into Equation 1, we get the equations of motion for the Linear Inverted Pendulum Plus Flywheel Model,

$$
\begin{aligned}
\ddot{x} & =\frac{g}{z_{0}} x-\frac{1}{m z_{0}} \tau_{h} \\
\ddot{\theta_{b}} & =\frac{1}{J} \tau_{h}
\end{aligned}
$$

Note that these equations of motion are linear, given that $\mathrm{z}$ is constant. This linearity is what makes the Linear Inverted Pendulum Model and the flywheel extension valuable as an analysis and design tool.

Before solving for the Capture Region for the Linear Inverted Pendulum Plus Flywheel Model, we first compute the Capture Point when the flywheel is not available.

\section{Capture Point for Linear Inverted Pendulum Model}

By setting $\tau_{h}=0$ we get the equation of motion for the Linear Inverted Pendulum Model:

$$
\ddot{x}=\frac{g}{z_{0}} x
$$

We can derive a conserved quantity called the "Linear Inverted Pendulum Orbital Energy" [3] by noting that this equation represents a mass-spring system with unit mass and a negative-rate spring with a stiffness of $-\frac{g}{z_{0}}$ :

$$
E_{L I P}=\frac{1}{2} \dot{x}^{2}-\frac{g}{2 z_{0}} x^{2}
$$

If the Center of Mass is moving toward the foot and $E_{L I P}>$ 0 , then there is enough energy for the CoM to go over the foot and continue on its way. If $E_{L I P}<0$, then the CoM will stop and reverse directions before getting over the foot. If $E_{L I P}=0$, then the CoM will come to rest over the foot. The equilibrium state $E_{L I P}=0$ defines the two eigenvectors of the system,

$$
\dot{x}= \pm x \sqrt{\frac{g}{z_{0}}}
$$

Equation 9 represents a saddle point with one stable and one unstable eigenvector. $x$ and $\dot{x}$ have opposite signs (the Center of Mass is moving toward the Center of Pressure) for the stable eigenvector and the same signs (the CoM is moving away from the $\mathrm{CoP}$ ) for the unstable eigenvector.

The Orbital Energy of the Linear Inverted Pendulum remains constant until the swing leg is placed and the feet change roles. Assuming that the exchange happens instantaneously, without energy loss, we can solve for foot placement based 
on either desired Orbital Energy, or desired speed at a given value of $x$ [3], [4]. For computing the Capture Point, we are interested in the foot placement required to obtain an Orbital Energy of zero and corresponding to the stable eigenvector from Equation 9,

$$
x_{\text {capture }}=\dot{x} \sqrt{\frac{z_{0}}{g}}
$$

For a given state the Linear Inverted Pendulum Model has a single Capture Point corresponding to the footstep that would put the state of the robot onto the stable eigenvector. When the flywheel is made available, this point will grow to a Capture Region. If the state is on one side of the stable eigenvector in phase space, then a clockwise acceleration of the flywheel will capture balance. If on the other side, then a counterclockwise acceleration will be required.

We first show two methods for computing an upper bound on the Capture Region assuming the flywheel can make either an instantaneous velocity change or an instantaneous position change. We then derive a more realistic Capture Region based on a torque-limited and angle-limited flywheel.

\section{Instantaneous Flywheel Velocity Change}

Suppose we produce an impulsive torque on the flywheel, that causes a step change in the rotational velocity of the flywheel of $\Delta \dot{\theta}_{b}$. Then we get a step change in the forward velocity of $\Delta \dot{x}=-\frac{J}{m z_{0}} \Delta \dot{\theta}_{b}$. In this case, the instantaneous Capture Region will be

$\sqrt{\frac{z_{0}}{g}}\left(\dot{x}-\frac{J}{m z_{0}} \Delta \dot{\theta}_{b_{\text {max }}}\right)<x_{\text {capture }}<\sqrt{\frac{z_{0}}{g}}\left(\dot{x}-\frac{J}{m z_{0}} \Delta \dot{\theta}_{b_{\text {min }}}\right)$

\section{E. Instantaneous Flywheel Position Change}

Suppose we could produce a step change in the rotational position of the flywheel of $\Delta \theta_{b}$. This would cause a step change in the position of the CoM of $\Delta x=-\frac{J}{m z_{0}} \Delta \theta_{b}$. In this case, the instantaneous Capture Region will be

$$
\sqrt{\frac{z_{0}}{g}} \dot{x}-\frac{J}{m z_{0}} \Delta \theta_{b_{\max }}<x_{\text {capture }}<\sqrt{\frac{z_{0}}{g}} \dot{x}-\frac{J}{m z_{0}} \Delta \theta_{b_{\text {min }}}
$$

\section{F. Torque and Angle Limited Linear Inverted Pendulum Plus Flywheel Capture Region}

Assuming step changes in either flywheel angular velocity or position results in easy to compute and potentially useful upper bounds on the Capture Region. However, such step changes are not physically possible. Here we assume the flywheel is torque limited and has limits on its minimum and maximum rotation angles.

Torque limits are realistic since most motors are torque limited and they can achieve the maximum torque nearly instantaneously when compared to physical time constants. By using step torque profiles, we can compute the Capture Region for the Linear Inverted Pendulum Plus Flywheel model fairly easily since the dynamics are linear and unit steps have simple Laplace Transforms.
Suppose the robot is moving at $\dot{x}_{0}$ and the flywheel is spinning at $\dot{\theta}_{0}$ and has an angle of $\theta_{0}$ with respect to vertical. We wish to find a flywheel torque profile and a stepping location that will bring the robot to rest over its foot with no forward velocity or flywheel angular velocity.

The torque profile that will provide the most influence on velocity is the one which accelerates the flywheel as hard as possible in one direction and then decelerates it, bringing it to a stop at the maximum flywheel angle,

$$
\tau(t)=\tau_{\max } u(t)-2 \tau_{\max } u\left(t-T_{R 1}\right)+\tau_{\max } u\left(t-T_{R 2}\right)
$$

where $\tau_{\max }$ is the maximum torque that the joint can apply, $u(t-T)$ is the unit step function starting at $T, T_{R 1}$ is the time at which the flywheel stops accelerating and starts decelerating and $T_{R 2}$ is the time at which the flywheel comes to a stop.

Given the torque profile in Equation 13, the flywheel angular velocity and position will be

$$
\begin{aligned}
\dot{\theta}(t)= & \dot{\theta}_{0}+\frac{\tau_{\max }}{J}\left(u^{1}(t)-2 u^{1}\left(t-T_{R 1}\right)+u^{1}\left(t-T_{R 2}\right)\right) \\
\theta(t)= & \theta_{0}+\dot{\theta}_{0} t+ \\
& \frac{\tau_{\max }}{J}\left(\frac{1}{2} u^{2}(t)-u^{2}\left(t-T_{R 1}\right)+\frac{1}{2} u^{2}\left(t-T_{R 2}\right)\right)
\end{aligned}
$$

At time $T_{R 2}$ we want $\dot{\theta}\left(T_{R 2}\right)=\dot{\theta}_{f}$ and $\theta\left(T_{R 2}\right)=\theta_{\max }$. To find the extents of the Capture Region, $\dot{\theta}_{f}=0$. However, we keep $\dot{\theta}_{f}$ in the equation since it may be desirable to have a final velocity which helps return the flywheel to the starting position. Solving for $T_{R 1}$ in Equation 16 we get

$$
T_{R 1}=\frac{1}{2} T_{R 2}+\frac{J}{2 \tau_{\max }}\left(\dot{\theta}_{f}-\dot{\theta}_{0}\right)
$$

Substituting $T_{R 1}$ into Equation 14 and rearranging, we get a quadratic equation in $T_{R 2}$,

$$
\begin{gathered}
{\left[\frac{\tau_{\max }}{4 J}\right] T_{R 2}^{2}+\left[\frac{1}{2}\left(\dot{\theta}_{f}+\dot{\theta}_{0}\right)\right] T_{R 2}+} \\
{\left[\theta_{0}-\theta_{\max }-\frac{J}{4 \tau_{\max }}\left(\dot{\theta}_{f}-\dot{\theta}_{0}\right)^{2}\right]=0}
\end{gathered}
$$

which can be solved for $T_{R 2}$. Note that if $\dot{\theta}_{f}=\dot{\theta}_{0}=0$, then $T_{R 1}=\frac{1}{2} T_{R 2}$ and

$$
T_{R 2}=\sqrt{\frac{4 J}{\tau_{\max }}\left(\theta_{\max }-\theta_{0}\right)}
$$

We can now determine the position and velocity trajectories of the mass by integrating the equations of motion for the Linear Inverted Pendulum Plus Flywheel model,

$$
\ddot{x}=\frac{g}{z_{0}} x-\frac{1}{m z_{0}} \tau_{h}
$$

Written in form of Laplace transforms, we have

$$
\frac{X(s)}{\tau(s)}=-\frac{1}{m z_{0}}\left(\frac{1}{s^{2}-w^{2}}\right)
$$


where $w=\sqrt{\frac{g}{z_{0}}}$. The zero input response of this system is

$$
\begin{aligned}
& x(t)_{Z I R}=x_{0} \cosh (w t)+\frac{1}{w} \dot{x}_{0} \sinh (w t) \\
& \dot{x}(t)_{Z I R}=w x_{0} \sinh (w t)+\dot{x}_{0} \cosh (w t)
\end{aligned}
$$

The zero state response, given the input in Equation 13 is

$$
\begin{aligned}
x(t)_{Z S R}=-\frac{\tau_{\max }}{m z_{0} w^{2}} & {[(\cosh (w t)-1) u(t)} \\
& -2\left(\cosh \left(w\left(t-T_{R 1}\right)\right)-1\right) u\left(t-T_{R 1}\right) \\
& \left.+\left(\cosh \left(w\left(t-T_{R 2}\right)\right)-1\right) u\left(t-T_{R 2}\right)\right]
\end{aligned}
$$

$$
\begin{aligned}
\dot{x}(t)_{Z S R}=-\frac{\tau_{\max }}{m z_{0} w} \quad & {[\sinh (w t) u(t)} \\
& -2 \sinh \left(w\left(t-T_{R 1}\right)\right) u\left(t-T_{R 1}\right) \\
& \left.+\sinh \left(w\left(t-T_{R 2}\right)\right) u\left(t-T_{R 2}\right)\right\}(22)
\end{aligned}
$$

Combining the zero input response and the zero state response at time $T_{R 2}$ we have

$$
\begin{aligned}
& x\left(T_{R 2}\right)=P_{1}+P_{2} x_{0} \\
& \dot{x}\left(T_{R 2}\right)=P_{3}+P_{4} x_{0}
\end{aligned}
$$

where

$$
\begin{aligned}
P_{1}= & \frac{1}{w} \dot{x}_{0} \sinh \left(w T_{R 2}\right) \\
& -\frac{\tau_{\max }}{m z_{0} w^{2}}\left[\cosh \left(w T_{R 2}\right)-2 \cosh \left(w\left(T_{R 2}-T_{R 1}\right)\right)+1\right] \\
P_{2}= & \cosh \left(w T_{R 2}\right) \\
P_{3}= & \dot{x}_{0} \cosh \left(w T_{R 2}\right) \\
& -\frac{\tau_{\max }}{m z_{0} w}\left[\sinh \left(w T_{R 2}\right)-2 \sinh \left(w\left(T_{R 2}-T_{R 1}\right)\right)\right] \\
P_{4}= & w \sinh \left(w T_{R 2}\right)
\end{aligned}
$$

To solve for a Capture Point, we need the state to lie on the stable eigenvector of the Linear Inverted Pendulum system after the flywheel stops at $T_{R 2}$,

$$
\dot{x}\left(T_{R 2}\right)=-w x\left(T_{R 2}\right)
$$

Using Equations 23 and 25 and the fact that $\cosh (y)+$ $\sinh (y)=e^{y}$, we can solve for $x_{0}$,

$$
x_{0}=-\frac{1}{w} \dot{x}_{0}+\frac{\tau_{\max }}{m g}\left[\frac{\left(e^{w T_{R 2}}-2 e^{w\left(T_{R 2}-T_{R 1}\right)}+1\right)}{e^{w T_{R 2}}}\right]
$$

The Capture Point is then $-x_{0}$. To find the other boundary of the Capture Region, the above can be repeated with the torque limit of $\tau_{\min }$ and the angle limit of $\theta_{\min }$. To find a Capture Point without the use of angular momentum, one can repeat the above, except set $T_{R 1}=0 . T_{R 2}$ will be long enough to stop any spin that the flywheel may currently have and $x_{0}$ can be solved as before.

\section{G. Ground Reaction Forces in Linear Inverted Pendulum Plus Flywheel Model}

The ground reaction forces in the Linear Inverted Pendulum Plus Flywheel model can be computed considering a free body diagram and examining $\ddot{x}$ and $\ddot{z}$ :

$$
f_{z}=m g, f_{x}=\frac{m g}{z_{0}} x-\frac{\tau_{h}}{z_{0}}
$$

To prevent slipping, the ground reaction force vector must stay within the friction cone. Given a coefficient of friction, $\alpha$,

$$
-\alpha<\frac{f_{x}}{f_{z}}=\frac{x}{z_{0}}-\frac{\tau_{h}}{m g z_{0}}<\alpha
$$

If $\tau_{h}=0$ then we get $\frac{f_{x}}{f_{z}}=\frac{x}{z_{0}}$, which means that the angle of the virtual leg from the Center of Mass to the Center of Pressure must be inside the friction cone. For nonzero $\tau_{h}$, the ground reaction force vector is rotated to produce this torque about the CoM. For typical coefficients of friction, the above equation gives us a limit on $\tau_{h}$, or equivalently on $\ddot{\theta_{b}}=\frac{\tau_{h}}{J}$. Note that a step change in either $\dot{\theta}_{b}$ or $\theta_{b}$, would require an impulsive torque, which would cause the ground reaction force to be horizontal, causing slipping on any non-attached surface.

\section{H. Dimensional Analysis}

We can perform a dimensional analysis [10] of the state variables and parameters of the Linear Inverted Pendulum plus Flywheel Model to reduce the number of variables involved. Let us define dimensionless position, velocity, time, inertia, torque, and angles as

$$
\begin{aligned}
x^{\prime} & \equiv \frac{x}{z_{0}} \\
\dot{x}^{\prime} & \equiv \frac{1}{\sqrt{g z_{0}}} \dot{x} \\
t^{\prime} & \equiv t \sqrt{\frac{g}{z_{0}}} \\
J^{\prime} & \equiv \frac{J}{m z_{0}^{2}} \\
\tau^{\prime} & \equiv \frac{\tau}{m g z_{0}} \\
\theta^{\prime} & \equiv J^{\prime} \theta
\end{aligned}
$$

Note that the dimensionless inertia can also be written as a ratio of the radius of gyration of the pendulum and the Center of Mass height, $J^{\prime} \equiv \frac{J}{m z_{0}^{2}}=\frac{R_{g y r}^{2}}{z_{0}^{2}}$. For a point mass, $J^{\prime}=0$, and for a flywheel with all of its mass on the rim, which just touches the ground, $J^{\prime}=1.0$.

With these dimensionless quantities, the equations of motion for the Linear Inverted Pendulum Plus Flywheel Model (Equation 6) become

$$
\begin{aligned}
& \ddot{x^{\prime}}=x^{\prime}-\tau^{\prime} \\
& \ddot{\theta}^{\prime}=\tau^{\prime}
\end{aligned}
$$

where time derivatives are with respect to non-dimensional time: $\ddot{x}^{\prime} \equiv \frac{d^{2} x^{\prime}}{d t^{\prime 2}}$ and $\ddot{\theta^{\prime}} \equiv \frac{d^{2} \theta^{\prime}}{d t^{\prime 2}}$. With this formulation, the only remaining parameters used in our derivation of Capture Regions are $\tau_{\text {max }}^{\prime}, \theta_{\text {max }}^{\prime}, \tau_{\text {min }}^{\prime}$, and $\theta_{\text {min }}^{\prime}$. Therefore, two Linear Inverted Flywheel Plus Pendulum systems are dynamically similar if they have the same values of those four quantities. In order to reformulate the equations for computing the Capture Region, one can replace all the variables with their dimensionless versions and set $m, g, J$ and $z_{0}$ all to one. For example, the ground reaction force limits become

$$
-\alpha<x^{\prime}-\tau^{\prime}<\alpha
$$




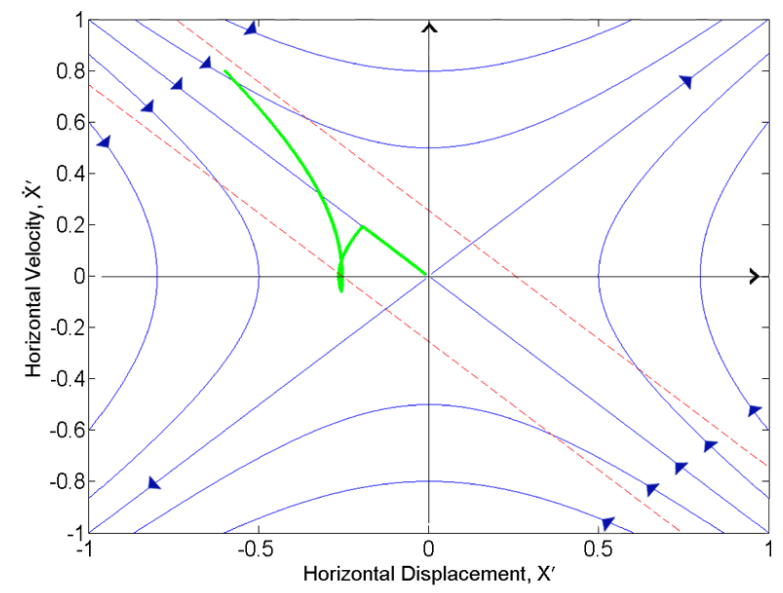

Fig. 3. Phase portrait of Linear Inverted Pendulum Model, with expanded Capture Region (dotted). The Figure is plotted using dimensionless quantities, with $\tau_{\max }^{\prime}=0.5, \theta_{\max }^{\prime}=\frac{\pi}{4}, \theta_{0}^{\prime}=0$, and $\dot{\theta}_{0}^{\prime}=0$. The green curve is a trajectory using angular momentum of the flywheel in order to stop, starting from initial conditions of $x_{0}^{\prime}=-0.6$ and $\dot{x}_{0}^{\prime}=0.8$.

\section{Vi. Phase Portrait and Parameter Variations}

In this Section we present phase portraits of the nondimensional system as well as figures showing how the Capture Region changes as the maximum torque or maximum rotation angle change. We assume that the robot starts with zero initial rotation angle and velocity $\left(\theta_{0}=0, \dot{\theta}_{0}=0\right)$. Therefore, the extents of the Capture Region can be solved using the non-dimensional version of Equation 26 with $T_{R 1}=$ $\frac{1}{2} T_{R 2}$,

$$
x_{0}^{\prime}=-\dot{x}_{0}^{\prime}+\tau_{\max }\left[\frac{\left(e^{T_{R 2}^{\prime}}-2 e^{\frac{1}{2} T_{R 2}^{\prime}}+1\right)}{e^{T_{R 2}^{\prime}}}\right]
$$

where

$$
T_{R 2}^{\prime}=2 \sqrt{\frac{\theta_{\max }^{\prime}-\theta_{0}^{\prime}}{\tau_{\max }^{\prime}}}
$$

Figure 3 shows the phase portrait of the Linear Inverted Pendulum Model in dimensionless coordinates, along with the expanded region of state space that the robot can stop from (dotted) resulting from the addition of angular momentum. For generating this figure we used $\tau_{\max }^{\prime}=0.5, \theta_{\max }^{\prime}=\frac{\pi}{4}$, $\tau_{\min }=-\tau_{\max }, \theta_{\min }=-\theta_{\max }, \theta_{0}^{\prime}=0$, and $\dot{\theta}_{0}^{\prime}=0$. Also shown is the resultant trajectory (green) for recovering balance with the aid of the flywheel for one set of initial conditions.

Figure 3 shows that with the flywheel, the robot can stop from a wide band of states centered about, and parallel to, the stable eigenvector line. Note the excursions of the example trajectory outside the Capture Region. This excursion is possible since the flywheel has a rotational velocity during this trajectory, whereas the extents of the Capture Region assume zero initial rotational velocity.

Figure 4 shows how the Capture Region varies as the maximum torque, $\tau_{\max }^{\prime}$, varies and Figure 5 shows how the Capture Region varies as the maximum rotation angle, $\theta_{\text {max }}^{\prime}$,

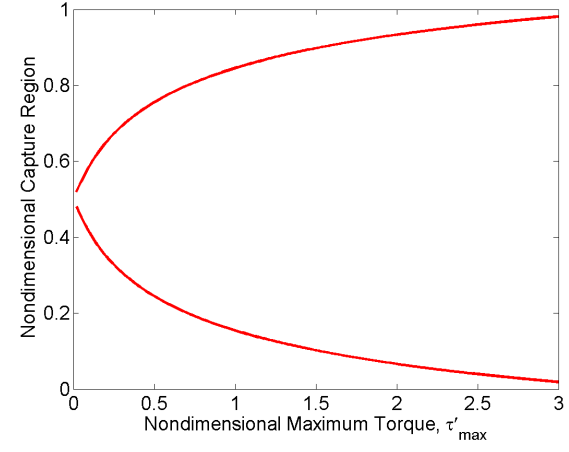

Fig. 4. Variation of the Capture Region for $\dot{x}_{0}^{\prime}=0.5$ as the maximum dimensionless torque varies from $\tau_{\max }^{\prime}=0.0$ to $\tau_{\max }^{\prime}=2.0$. Figure plotted using dimensionless quantities, with $\theta_{\max }^{\prime}=\frac{\pi}{4}, \theta_{0}^{\prime}=0$, and $\dot{\theta}_{0}^{\prime}=0$.

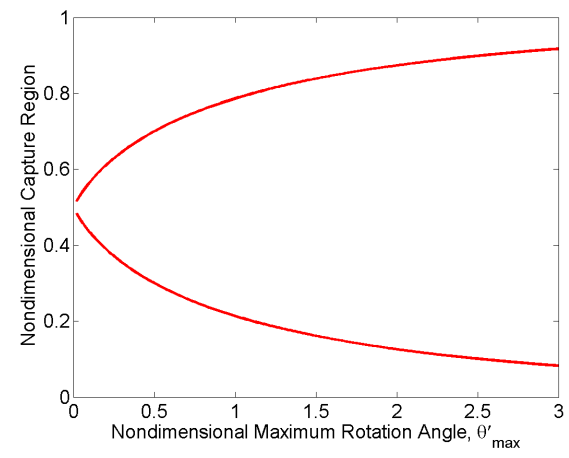

Fig. 5. Variation of the Capture Region for $\dot{x}_{0}^{\prime}=0.5$ as the maximum dimensionless flywheel angle varies from $\theta_{\max }^{\prime}=0.0$ to $\theta_{\max }^{\prime}=3.0$. Figure plotted using dimensionless quantities, with $\tau_{\max }^{\prime}=0.5, \theta_{0}^{\prime}=0$, and $\dot{\theta_{0}^{\prime}}=0$.

varies. For both of these figures $\dot{x}_{0}^{\prime}=0.5, \tau_{\min }^{\prime}=-\tau_{\max }^{\prime}$, $\theta_{\text {min }}^{\prime}=-\theta_{\text {max }}^{\prime}, \theta_{0}^{\prime}=0$, and $\dot{\theta}_{0}^{\prime}=0$. For Figure $4, \theta_{\text {max }}^{\prime}=\frac{\pi}{4}$. For Figure 5, $\tau_{\max }^{\prime}=0.5$.

Both Figure 4 and Figure 5 reach asymptotic limits as $\tau_{\max }^{\prime}$ or as $\theta_{\max }^{\prime}$ approaches infinity. We can solve for these limits using Equations 38 and 39 and L'Hospital's rule. The results are

$$
\begin{gathered}
\lim _{\tau_{\text {max }}^{\prime} \rightarrow \infty} x_{0}^{\prime}=-\dot{x}_{0}^{\prime}+\theta_{\text {max }}^{\prime}-\theta_{0}^{\prime} \\
\lim _{\theta_{\max }^{\prime} \rightarrow \infty} x_{0}^{\prime}=-\dot{x}_{0}^{\prime}+\tau_{\text {max }}^{\prime}
\end{gathered}
$$

Note that the first of these matches the result in Equation 12 where we assumed we could make an instantaneous angular position change of the flywheel. That would be the case as the maximum torque approaches infinity.

\section{Simulation Results}

We performed simulations on a simple, four degree of freedom, bipedal walking model using the Yobotics Simulation Construction Set. The two legs have rotating hip joints, extensional knee joints, and point feet. The center of mass of the body is at the hip joint and is represented as a flywheel with mass of $m=25 \mathrm{~kg}$ and inertia of $J=1.225 \mathrm{kgm}^{2}$. The legs 


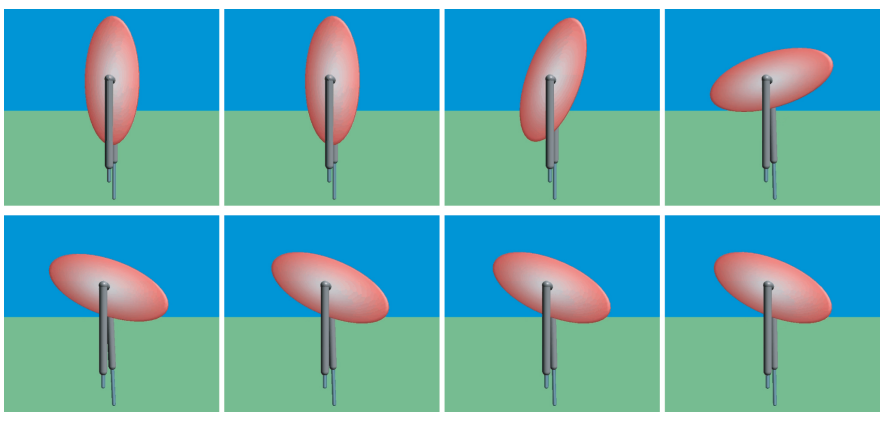

Fig. 6. Time elapsed image sequence showing the simulated robot recover from a disturbance by lunging its body. Snapshots are left to right, top to bottom, and are taken at 0.1 second increments.

are very light compared to the body, modeled as point masses of $0.0025 \mathrm{~kg}$ at each foot. The maximum flywheel angle is $\theta_{\max }=\frac{3}{4} \pi$ and the maximum hip torque is $\tau_{\max }=100 \mathrm{Nm}$. The desired Center of Mass height is $z_{0}=0.9375 \mathrm{~m}$. With these values, the non-dimensional parameters are $J^{\prime}=0.0558$, $\tau_{\text {max }}^{\prime}=0.435, \theta_{\text {max }}^{\prime}=0.131$.

Figure 6 shows a time elapsed image sequence after an impulsive disturbance that changes the velocity from rest to $0.2 \mathrm{~m} / \mathrm{s}$. Figure 7 shows the corresponding data. The disturbance occurs at $0.5 \mathrm{~s}$ and the lunge is completed by approximately $0.8 s$.

Figure 8 shows a time elapsed image sequence of the robot walking with a top-of-stride velocity of $0.5 \mathrm{~m} / \mathrm{s}$ and then stopping in a single step by lunging. Figure 9 shows the corresponding data. The command to stop is given at approximately $3.9 \mathrm{~s}$ and the lunge is completed at approximately $4.25 \mathrm{~s}$.

In both of these simulations, we were able to have nearly zero orbital energy (to within three decimal places) after the lunge, thereby providing validation to our theoretical analysis. Discrepancies from exactly 0.0 orbital energy can be attributed to the small but finite mass in the feet, the compliance of the simulated ground, numerical discretization and roundoff effects.

\section{DISCUSSION AND FUTURE WORK}

\section{A. Application to More Complex Humanoids}

All of the equations presented in this paper are exact for the Linear Inverted Pendulum Plus Flywheel Model. How well they will work as approximations for controlling a nonsimulated three-dimensional humanoid with distributed mass that does not walk at a constant height needs to be determined. Ongoing work is directed at relaxing the constant height constraint, empirically determining the effects of model discrepancies by examining more complex models, and applying our results to a real bipedal robot.

Note that exact computations for a general humanoid may not be required and the approximations used in this paper may be acceptable. As long as approximations of Capture Points can be kept on the order of the size of a humanoid foot, the ability to move the Center of Pressure inside the foot may be able to make up for errors due to the use of approximate
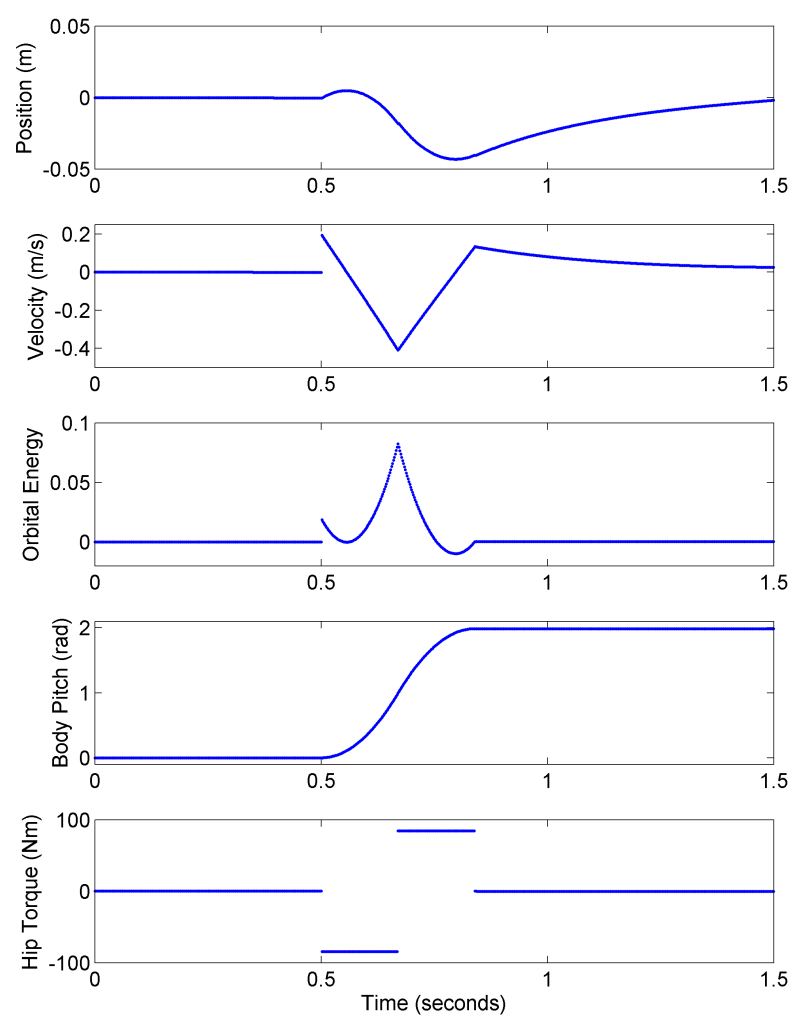

Fig. 7. Simulation data corresponding to Figure 6 . The robot is impulsively pushed at $0.5 \mathrm{sec}$., increasing its velocity to $0.2 \mathrm{~m} / \mathrm{s}$. A body lunge recovers balance without requiring a step.

models. Also, in many cases it is not critical to stop in one step. Small errors in Capture Point locations may simply require two or three steps to stop, when one step could have been possible.

The approximations presented in this paper can also be used as seed values if learning or adaptation techniques are used to automatically determine where to step and how to lunge in order to stop after a disturbance. Using seed values promises to result in faster learning than pure "black box" approaches.

\section{B. Under-Constrained Lunge}

In both of the simulations presented, the maximum hip torque of $100 \mathrm{Nm}$ and the maximum body pitch of $\frac{3}{4} \pi$ were not required to stop the robot. In other words, in both cases, the foot was inside the Capture Region before the lunge took place. Therefore an infinity of torque profiles could have been used to stop. We chose to use a bang-bang profile in which $T_{R 2}$ was determined assuming full torque and rotation angle were required. Then we solved for the actual step torque profile that results in stopping the robot, given the value of $T_{R 2}$. Alternatively, we could have used the maximum torque and solved for the lunge time and rotation angle, or used the maximum rotation angle and solved for the torque and lunge time. Which of these or other lunge method is used could be determined through many techniques, including solving an optimization problem, and is an area that needs to be explored. 

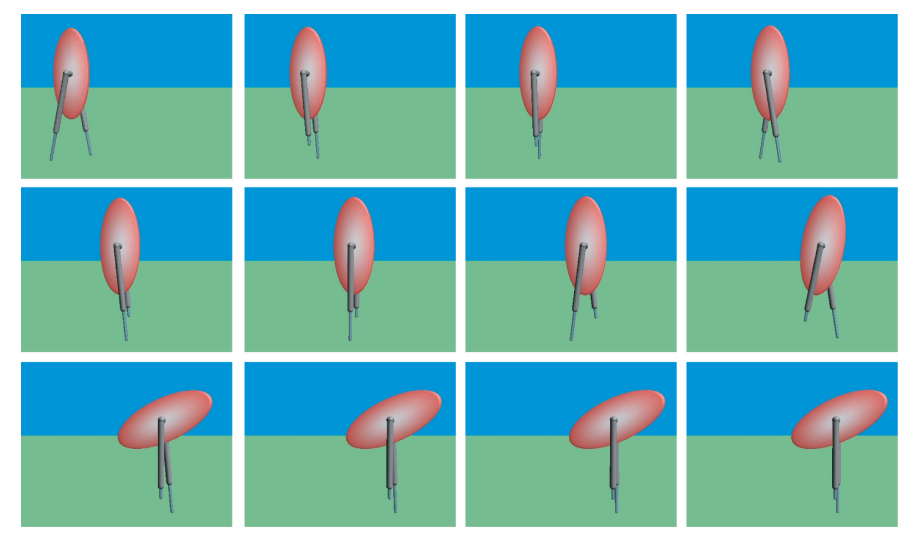

Fig. 8. Time elapsed image sequence showing the robot stop walking in a single step by lunging its body. Snapshots are left to right, top to bottom, and are taken at 0.25 second increments.

\section{Capture Region and Biped Design Considerations}

Increasing the amount of available non-dimensional torque, $\tau_{\text {max }}^{\prime}$, or rotation angle, $\theta_{\text {max }}^{\prime}$, increases the size of the Capture Region. However, if one of the values is fixed, there is diminishing returns in increasing the other, as seen in Figures 4 and 5. In addition, the maximum torque must not exceed the value that would cause foot slipping.

Therefore, these two parameters should be designed together, using Equations 38 to 41 to analyze their effect on the Capture Region. A feasible approach would be to use Equation 37 to set a good maximum torque and then increase the maximum rotation angle until there are diminishing returns. Determining how maximizing the Capture Region fits in with other design criterion needs to be explored further.

\section{ACKNOWLEDGMENT}

Support for this work was provided by the Honda Research Institute.

\section{REFERENCES}

[1] Muhammad Abdallah and Ambarish Goswami. A biomechanically motivated two-phase strategy for biped upright balance control. IEEE International Conference on Robotics and Automation (ICRA), 2005.

[2] Andreas G. Hofmann. Robust Execution of Bipedal Walking Tasks From Biomechanical Priciples. PhD thesis, Computer Science Department, Massachusetts Institute of Technology, 2006.

[3] S. Kajita and K. Tani. Study of dynamic biped locomotion on rugged terrain-derivation and application of the linear inverted pendulum mode. volume 2, pages 1405 - 1411. IEEE International Conference on Robotics and Automation (ICRA), 1991.

[4] Shuuji Kajita, Fumio Kanehiro, Kenji Kaneko, Kazuhito Yokoi, and Hirohisa Hirukawa. The $3 \mathrm{~d}$ linear inverted pendulum mode: a simple modeling for a biped walking pattern generation. pages $239-246$. IEEE International Conference on Intelligent Robots and Systems (IROS), 2001.

[5] S. Kajita, F. Kanehiro, K. Kaneko, K. Fujiwara, K. Harada, K. Yokoi, and H. Hirukawa, "Resolved momentum control: Humanoid motion planning based on the linear and angular momentum," in IEEE/RSJ International Conference on Intelligent Robots and Systems, 2003, pp. 1644-1650.

[6] T. Komura, H. Leung, S. Kudoh, and J. Kuffner, "A feedback controller for biped humanoids that can counteract large perturbations during gait," in IEEE International Conference on Robotics and Automation (ICRA), 2005, Barcelona, Spain, pp. 2001-2007.

[7] T. Komura, A. Nagano, H. Leung, and Y. Shinagawa, "Simulating pathological gait using the enhanced linear inverted pendulum model," IEEE Transactions on Biomedical Engineering, vol. 52, no. 9, pp. 15021513, 2005, September.
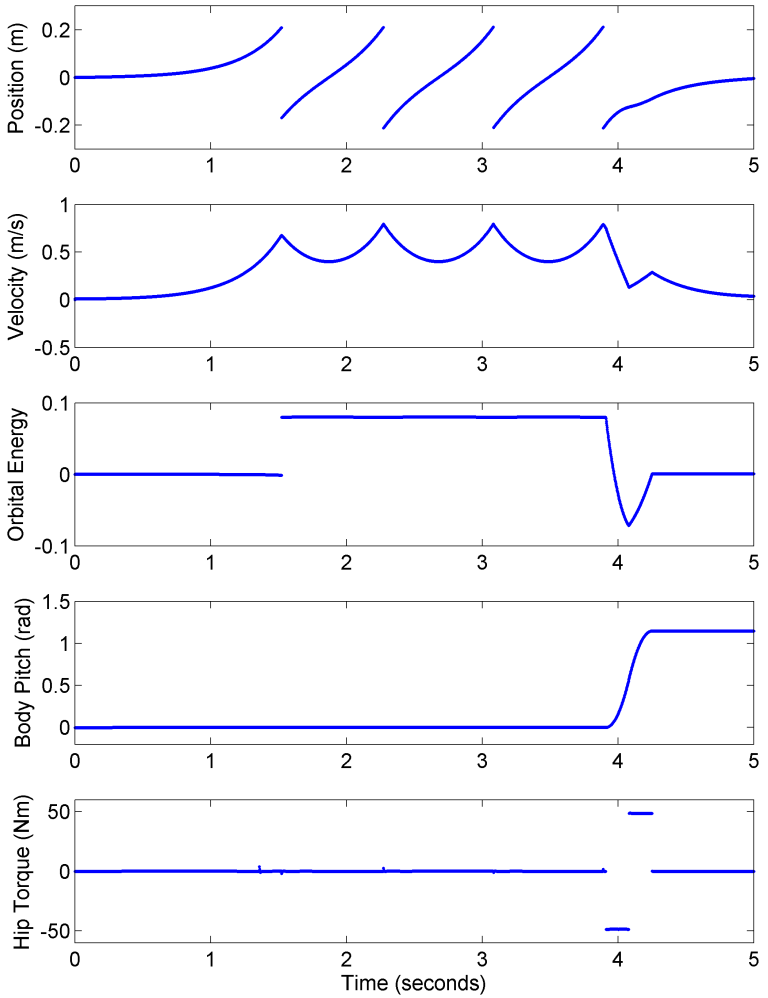

Fig. 9. Simulation data corresponding to Figure 8. The robot starts walking at a desired top-of-stride velocity of $0.5 \mathrm{~m} / \mathrm{s}$. The robot is commanded to stop shortly after taking a step at about 3.9 seconds. It does so by lunging its upper body, without taking an additional step.

[8] A.D. Kuo and F. E. Zajac. Human standing posture: multijoint movement strategies based on biomechanical constraints. Progress in Brain Research, 97:349-358, 1993.

[9] N. M. Mayer, F. Farkas, and M. Asada, "Balanced walking and rapid movements in a biped robot by using a symmetric rotor and a brake," in International Conference on Mechatronics and Automation, July 29August 1, 2005, Niagara Falls, Ontario, Canada.

[10] Thomas A. McMahon and John Tyler Bonner. On Size and Life. Scientific American Library, 1983.

[11] Marko Popovic, Amy Englehart, and Hugh Herr. Angular momentum primitives for human walking: Biomechanics and control. In Proceedings of the IEEE/RSJ International Conference on Intelligent Robots and Systems, 2004.

[12] Marko Popovic, Ambarish Goswami, and Hugh Herr. Ground reference points in legged locomotion: Definitions, biological trajectories and control implications. International Journal of Robotics Research, 24(12):1013-1032, Dec 2005.

[13] Jerry Pratt. Exploiting Inherent Robustness and Natural Dynamics in the Control of Bipedal Walking Robots. PhD thesis, Computer Science Department, Massachusetts Institute of Technology, 2000.

[14] Jerry Pratt and Russ Tedrake. Velocity Based Stability Margins for Fast Bipedal Walking. First Ruperto Carola Symposium in the International Science Forum of the University of Heidelberg entitled "Fast Motions in Biomechanics and Robots" Heidelberg Germany, September 7-9, 2005.

[15] M. J. Sidi, Spacecraft Dynamics and Control. NewYork, NY: Cambridge University Press, 1997.

[16] P. Tsiotras and H. Shen, "Satellite attitude control and power tracking with energy/momentum wheels," Jornal of Guidance, Control and Dynamics, vol. 24, no. 1, pp. 23-34, 2001.

[17] J. Vermeulen, B. Verrelst, B. Vanderborght, D. Lefeber, and P. Guillaume, "Trajectory planning for the walking biped."

[18] E. R. Westervelt, J. W. Grizzle, and D. E. Koditschek. Zero dynamics of underactuated planar biped walkers. IFAC-2002, Barcelona, Spain, pages 1-6, Jul 2002. 